\title{
Inverse heat transfer for optimization and on-line thermal properties estimation in composites curing
}

\author{
A. A. Skordos and I. K. Partridge \\ Advanced Materials Department, Cranfield University \\ Cranfield, Bedford, United Kingdom
}

\begin{abstract}
This paper presents the development and application of a heat transfer inversion procedure to the cure of thermoset based composites based on genetic algorithms. The procedure is utilized for process optimization applied to the curing of carbon fiber reinforced composites. The optimization objective is the selection of an appropriate cure schedule so that the duration of the curing is minimized subject to constraints related to the thermal gradients developed during the cure.

An alternative use of inversion concerns the integration of monitoring signals with modeling. Inversion is utilized to alter on-line the thermal properties used in the direct model so that monitoring results coincide with simulation predictions. This procedure is applied to the curing of a carbon fiber reinforced thermoset based composite, using thermal conductivity as the variable thermal property.
\end{abstract}

Keywords: Composites manufacturing; Optimization; Parameter estimation; Genetic algorithms 


\section{INTRODUCTION}

In recent years the need for predictive modeling and for in-situ real time monitoring of composites manufacturing processes has arisen and been met by the development of a family of appropriate techniques. Models representing various aspects of processing have been developed and applied to the majority of processing techniques. Heat transfer models have been implemented in order to simulate the curing phenomena in autoclave processing [1], resin transfer molding [2], pultrusion [3] and filament winding [4]. Provided that these models are combined with appropriate cure kinetics subroutines [5,6], they offer the ability to calculate the spatial distributions of temperature and of the degree-of-cure and their evolution with time during the curing. Alongside with simulation, process monitoring methods such as thermal [7], dielectric [8,9], fiber optic [10] and acoustic cure monitoring [11], have begun to be implemented in an industrial environment.

Both monitoring and modeling are valuable for optimizing the curing process. The predictive ability of the simulation can be used as a part of the process design, while monitoring constitutes a potential tool for on line control. However, both approaches present some inherent drawbacks. Accurate modeling requires an extensive knowledge of material properties and process characteristics. This may be impossible in some cases due to limited reproducibility of some of the process conditions, or due to the prohibitive costs associated with the knowledge acquisition step. Similarly, monitoring involves insertion of a sensor in some critical area of the component, which composite manufacturers and end users are reluctant to adopt.

A method to overcome these limitations arises from a combination of modeling and monitoring. In the present paper a scheme which combines heat transfer modeling and cure monitoring is presented. An inversion of the heat transfer model based on a genetic algorithm is applied to data gathered by monitoring, in order to calculate some of the properties. Thus, an estimation of those modeling parameters that are most difficult to predefine can be performed, in accordance with the results 
of monitoring. Subsequently the direct model can be solved in order to obtain the global picture of the cure.

The inversion procedure developed here is also applied to purely predictive process design where the minimization of the cure duration is the objective of the optimization.

\section{DIRECT MODEL}

\section{Heat Transfer Problem}

The model concerns the curing of a carbon fiber reinforced composite in a resin transfer mold. In this process dry fabric is placed in a rigid cavity, resin is infused under pressure and vacuum and the curing takes place with further heating of the mold. When forced convection does not occur heat conduction is the only heat transfer mechanism relevant to composites cure. Accordingly, the governing equation is:

$$
\rho c_{p} \frac{\partial T}{\partial t}=\nabla \cdot \boldsymbol{K} \nabla T+\left(1-v_{f}\right) \rho_{r} H_{t o t} \frac{d \alpha}{d t}
$$

The second term in the right side of (1) expresses the heat generated by the curing reaction. This equation is accompanied by a set of boundary conditions. In the general case there are three possible boundary conditions: -(i) prescribed temperature:

$$
T(\hat{\boldsymbol{r}}, t)=T^{\prime}(\hat{\boldsymbol{r}}, t), \quad \hat{\boldsymbol{r}} \in S_{1}
$$

(ii) prescribed heat flux

$$
\hat{\boldsymbol{n}} \cdot \boldsymbol{K} \nabla T(\hat{\boldsymbol{r}}, t)=q^{\prime}(\hat{\boldsymbol{r}}, t), \quad \hat{\boldsymbol{r}} \in S_{2}
$$

(iii) and convection

$$
\hat{\boldsymbol{n}} \cdot \boldsymbol{K} \nabla T(\hat{\boldsymbol{r}}, t)=-h\left(T(\hat{\boldsymbol{r}}, t)-T_{\infty}\right), \quad \hat{\boldsymbol{r}} \in S_{3}
$$

As in composites curing the heat transfer problem is multi-material, i. e. thermal properties and especially thermal conductivity present a discontinuity at the toolcomposite interface, a separate boundary value problem, of the type expressed by 
Eqs. (1)-(4), is formed over each sub-domain. An additional set of interfacial conditions that ensures temperature and heat flux continuity is defined as follows:

$$
\begin{aligned}
& T_{i}(\hat{\boldsymbol{r}}, t)=T_{j}(\hat{\boldsymbol{r}}, t), \quad \hat{\boldsymbol{r}} \in \widetilde{S}_{i j} \\
& \hat{\boldsymbol{n}} \cdot \boldsymbol{K}_{\boldsymbol{i}} \nabla T_{i}(\hat{\boldsymbol{r}}, t)=\hat{\boldsymbol{n}} \cdot \boldsymbol{K}_{j} \nabla T_{j}(\hat{\boldsymbol{r}}, t), \quad \hat{\boldsymbol{r}} \in \widetilde{S}_{i j}
\end{aligned}
$$

here the indices $i$ and $j$ denote areas of different materials.

Eqs. (1)-(6), accompanied by an appropriate cure kinetics model and a set of thermal properties models expressing thermal conductivity, specific heat capacity and density as functions of degree of cure and temperature, suffice for the complete description of the curing process in a resin transfer mold.

\section{Finite Elements Formulation}

In order to solve the problem using finite elements the domain is divided into a number of elements that connect at $G$ nodal points. The unknown variable is approximated as a linear combination of a set of $\mathrm{G}$ functions as follows:

$$
\bar{T}=\sum_{i=1}^{G} T_{i}(t) N_{i}(\hat{\boldsymbol{r}}), \quad T_{i}(t)=T^{\prime}\left(\boldsymbol{r}^{i}, t\right) \text { if } \boldsymbol{r}^{i} \in S_{1}
$$

Function $N_{i}$ is equal to unity at node $\mathrm{i}$ and vanishes at all other nodes and within all the elements to which node i does not connect.

By employing the above approximation, adopting a finite difference scheme to express time derivatives and expressing the heat transfer problem by its weighted residuals equivalent, the following system of equations is obtained:

$$
(\boldsymbol{M}+\theta \Delta t \boldsymbol{L}) \boldsymbol{T}^{n+1}-(\boldsymbol{M}-(1-\theta) \Delta t \boldsymbol{L}) \boldsymbol{T}^{n}-\Delta t \boldsymbol{F}=0
$$

where

$$
\begin{aligned}
& M_{j i}=\int_{\Omega} \rho c_{p} N_{j} N_{i} d \Omega \\
& L_{j i}=\int_{\Omega} \nabla N_{j} \cdot \boldsymbol{K} \nabla N_{i} d \Omega+\int_{S_{3}} N_{j} h N_{i} d S \\
& F_{j}=-\int_{S_{2}} N_{j} q^{\prime} d S+\int_{S_{3}} N_{j} h T_{\infty} d S+\int_{\Omega} N_{j}\left(l-v_{f}\right) \rho_{r} H_{t o t} \frac{d \alpha}{d t} d \Omega
\end{aligned}
$$


The system of equations described by (8)-(11) can be solved for each time step in order to calculate the distributions of temperature and degree of cure and their evolution with time.

\section{Model Implementation and Validation}

A model based on the principles described previously was developed in order to simulate the curing stage of resin transfer molding. The model comprises a core finite elements solver and a set of sub-models simulating the cure reaction kinetics, and the changes in thermal properties, i.e. specific heat capacity, thermal conductivity and density, during the cure. The algorithm starts from the initial temperature and degree of cure distributions which are fed into the cure kinetics model. The cure kinetics model produces values for the reaction rate which are fed into the finite elements solver in order to account for heat generation and values for the updated degree of cure which are output to the thermal properties sub-models. The three thermal properties sub-models use the values of conversion and initial temperature to compute the values of the thermal properties within the different elements of the model. The results are sent to the finite elements solver, which, taking into account the boundary conditions and the initial temperature distributions, computes the resulting temperature distribution. This procedure is repeated for a number of time steps by replacing the initial conditions with the temperature output of the finite elements model and the updated degree of cure distributions as calculated by the cure kinetics and by updating the boundary conditions.

The model was applied to the curing of an RTM6 epoxy resin/ T300 continuous carbon fiber reinforced composite. The material properties sub-models were appropriate to the specific materials. The cure kinetics model operates by direct interpolation in the degree of cure-temperature phase space applied to experimental differential scanning calorimetry (DSC) data and is analyzed in detail elsewhere [5].

The specific heat capacity sub-model operates in a similar way using experimental data produced by modulated differential scanning calorimetry. The 
experimental data for the resin in the temperature-fractional conversion phase space are illustrated in Fig. 1. The step change occurring during the cure of the resin marks the glass transition of the thermosetting material. The specific heat capacity of the carbon fiber was found to be a linear function of the temperature as follows:

$$
c_{p}=0.0023 T+0.765
$$

In the above equation the units of temperature are ${ }^{\circ} \mathrm{C}$ and of specific heat capacity $\mathrm{J} / \mathrm{g} /{ }^{\circ} \mathrm{C}$. Once the values corresponding to the resin and the reinforcement have been computed by the sub-model the law of mixtures is employed to calculate the value for the composite.

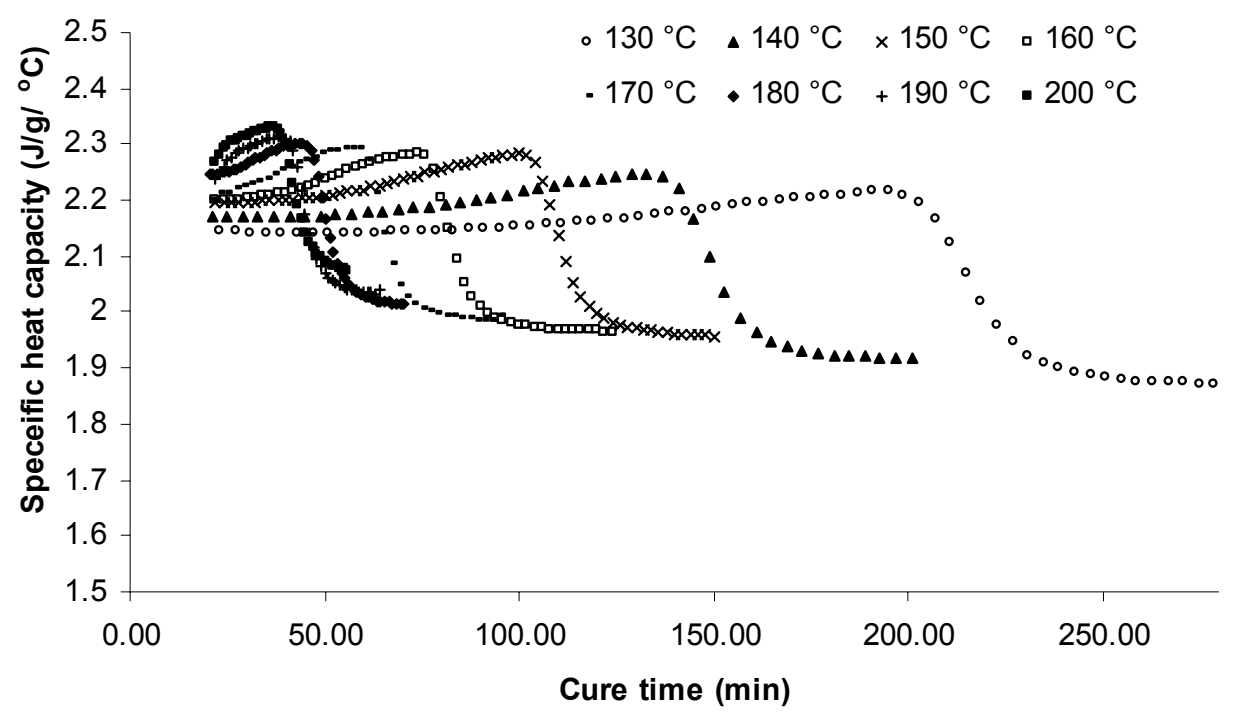

FIGURE 1 Specific heat capacity versus cure time during isothermal cures

The thermal conductivity of the anisotropic composite material is computed using an appropriate geometry based model [12] that combines values for the resin and the carbon fiber. The values for the resin were obtained experimentally using a technique that measures the thermal conductivity of the thermoset while it cures. Details of the method are given elsewhere [13]. The dependence of the resin thermal conductivity to temperature and fractional conversion can be expressed as follows: 


$$
K=0.0008 T \alpha^{2}-0.0011 T \alpha-0.0002 T-0.0937 \alpha^{2}+0.22 \alpha+0.12
$$

where temperature is given in ${ }^{\circ} \mathrm{C}$ and thermal conductivity in $\mathrm{W} / \mathrm{m} /{ }^{\circ} \mathrm{C}$. The longitudinal thermal conductivity of T300 carbon fiber can be expressed as follows [14]:

$$
K=4.8+0.0074 T
$$

where temperature is given in ${ }^{\circ} \mathrm{C}$ and thermal conductivity in $\mathrm{W} / \mathrm{m} /{ }^{\circ} \mathrm{C}$. The radial thermal conductivity of T300 carbon fiber is $0.84 \mathrm{~W} / \mathrm{m} /{ }^{\circ} \mathrm{C}$.

The density model takes into account thermal expansion of the resin and of the fiber and curing shrinkage. The model assumes that resin shrinkage is proportional to the progress of the curing reaction and uses the law of mixtures in order to calculate the composite density. The density of uncured RTM6 resin at ambient temperature is $1.117 \mathrm{~g} / \mathrm{cm}^{3}$ and the total volumetric chemical shrinkage $4.9 \%$, while the volumetric thermal expansion coefficient above glass transition is $4.08 \times 10^{-4}{ }^{\circ} \mathrm{C}^{-1}$ and below glass transition $1.62 \times 10^{-4}{ }^{\circ} \mathrm{C}^{-1}$ [15]. The expansion coefficient of carbon $\mathrm{T} 300$ is $5 \mathrm{x}$ $10^{-6}{ }^{\circ} \mathrm{C}^{-1}$ and its density at ambient temperature $1.8 \mathrm{~g} / \mathrm{cm}^{3}$.

The model implementation was tested against experimental data obtained during the cure of a composite. The experimental equipment used is shown in Fig.2. The dimensions of the mold cavity were $800 \mathrm{~mm} \times 340 \mathrm{~mm} \times 3 \mathrm{~mm}$. The sides of the cavity were sealed using silicone rubber while the tool was closed using a glass plate and a set of stiffeners. Heating is achieved by an array of heating elements placed under the mold cavity. The specific experimental configuration was selected in order to reduce the heat transfer problem to one dimension. This enables an easier implementation of the inversion that follows to be made. The carbon fabric used had a surface density of $816 \mathrm{~g} / \mathrm{m}^{2}$ and comprised three layers of unidirectional fiber tows at angles $+45^{\circ},-45^{\circ}$ and $0^{\circ}$. Four layers of this fabric were used in the cavity to achieve a fiber weight fraction of 0.69 . The total sequence of unidirectional tow plies was $[+45 /-45 / 0 / 0 /-45 /+45]_{2 \mathrm{~S}}$. Resin filling was carried out at $120{ }^{\circ} \mathrm{C}$. After completion of the filling, heating at $1.5^{\circ} \mathrm{C} / \mathrm{min}$ was performed up to $160{ }^{\circ} \mathrm{C}$, and then 
the temperature was kept constant. Two thermocouples (k-type) which measure the temperature at the top of the composite and at the mid-thickness were placed in the center of the curing component.

The modeling domain considered comprised the composite and the glass top plate. The bottom of the composite was considered to follow the thermal profile measured by the tool temperature sensor. The rubber seal was assumed to act as an insulator (zero heat flux) on the sides of the composite component. Natural air convection was considered on the top and sides of the glass plate. The initial condition was considered to be zero fractional conversion and uniform temperature after the end of filling. The thermal properties of the glass plate are given in table 1 .

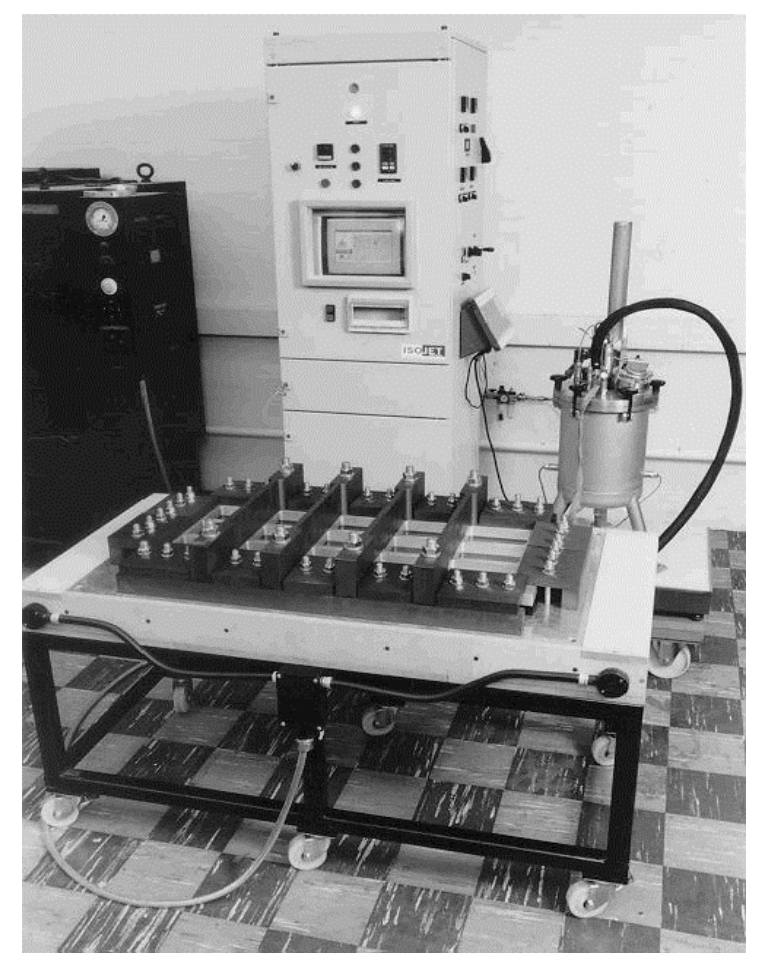

FIGURE 2 The resin transfer molding facility 
Table 1. Properties of the glass top plate

\begin{tabular}{c|c} 
Specific heat capacity $\left(\mathrm{J} / \mathrm{g} /{ }^{\circ} \mathrm{C}\right)$ & 0.84 \\
\hline Thermal conductivity $\left(\mathrm{W} / \mathrm{m} /{ }^{\circ} \mathrm{C}\right)$ & 0.78 \\
\hline Denisty $\mathrm{g} / \mathrm{cm}^{3} \rho\left(\mathrm{g} / \mathrm{cm}^{3}\right)$ & 2.7 \\
\hline Heat transfer coefficient $\left(\mathrm{W} / \mathrm{m}^{2} /{ }^{\circ} \mathrm{C}\right)$ & 8.5
\end{tabular}

The convergence of the three dimensional simulation was investigated. The convergence study indicated an optimum time step of $45 \mathrm{sec}$, an optimum element size of $0.05 \mathrm{~mm}$ in the thickness direction and $20 \mathrm{~mm}$ in the length and width directions of the component. A comparison of the results of the three dimensional case with the results of an one dimensional version of the model where only the thickness direction is considered showed that the 1-D model represents the curing of the specific component adequately. The results of the one dimensional model are set against experimental results in Fig. 3. It can be observed that the agreement achieved is satisfactory.

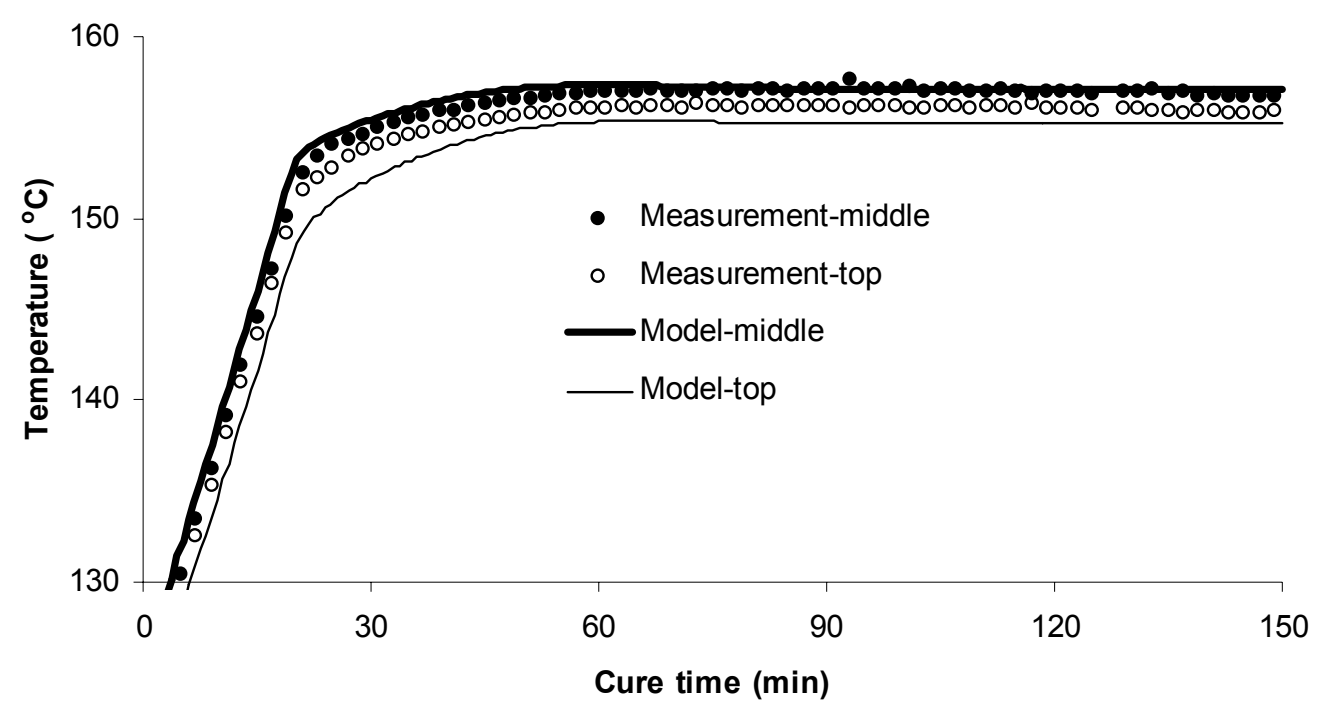

FIGURE 3 Experimental and simulation temperature 


\section{INVERSION PROCEDURE}

The inversion of the heat transfer model was performed using a genetic algorithm. The term describes a family of evolutionary optimization methods which involve a population of points in the search space of solutions (generation) and employ a performance sensitive selection procedure and crossover and mutation operations in order to reproduce a new population. The members of the population (individuals) are usually encoded in bit strings and the algorithm is iterated until some convergence criteria are met. A code which performs these procedures and uses as a direct model the one dimensional heat transfer simulation presented previously has been implemented. The operation of the algorithm is as follows.

A number $(\mathrm{N})$ of initial values for each parameter corresponding to the first generation are created using a random number generator. Then the 1-D simulation is executed $\mathrm{N}$ times and the results corresponding to each individual are stored in a file. The fitness of the individuals is calculated subsequently by comparing the output of the direct model with a target, which drives the inversion. The form of the fitness function and the inversion target are specific to the application of the inversion procedure.

The next step of the algorithm is the encoding of the individuals. In this stage, each individual is translated into a unique binary string. The length of the string defines the accuracy of the algorithm. Subsequently, the individuals are sorted according to their fitness. A limited number (m) of individuals with the best fitness are passed directly into the new generation. The rest of the individuals of the new generation are produced with a combination of selection, crossover and mutation. The selection is performed using a standard procedure called "roulette wheel". In this procedure each individual is assigned a slice of a circular wheel, the size of the slice being proportional to the fitness of the individual. Two random numbers between 0 and 360 are generated and the individuals corresponding to them are selected. The application of a uniform crossover operation to the two selected individuals follows. In this operation a predefined probability (exchange probability) is compared with a 
random number between 0 and 1 at each bit of the binary string. If the number is greater than the exchange probability the two selected individuals exchange their bit values, otherwise the values are preserved. At the end of this operation two new individuals have been produced, each of them containing parts of the old individuals. Subsequently, a mutation operation is applied to the two new individuals. In this stage a very low probability (mutation probability) is compared with a random number for each bit of the two new strings. If the mutation probability is greater than the random number, the bit of the string switches from 1 to 0 or from 0 to 1 , otherwise it remains unchanged.

When N-m individuals have been produced, the selection-reproduction procedure stops. These $\mathrm{N}-\mathrm{m}$ individuals together with the $\mathrm{m}$ best individuals of the previous generation form the new generation and the individuals are decoded back to decimal parameters.

At that point the convergence of the algorithm is tested according to a criterion specific to the application. If convergence has been reached, the algorithm outputs the appropriate data and exits. Otherwise the execution of the direct heat transfer model for the new individuals is performed and the whole procedure of fitness calculation, sorting, encoding, selection, reproduction and decoding is iterated until convergence is achieved.

\section{THERMAL PROFILE OPTIMIZATION}

A straightforward application of the inversion procedure described previously is the optimization of the thermal profile applied during the cure. The thermal profile comprises a linear heating up and an isothermal segment, thus can be characterized by two parameters:- (i) the ramp up rate and (ii) the isothermal temperature which are the subject of the optimization. The fitness function was selected so that it rewards the parameter values which reduce the duration of the curing stage, i.e. it increases as the time to reach a fractional conversion of 0.84 in all elements of the component decreases. This is implemented by the function: 


$$
\text { Fitness }=1 / t_{c}
$$

which is subject to the constraint:

$$
\left|\frac{d T}{d z}\right|_{\max }<2.5^{\circ} \mathrm{C} / \mathrm{mm}, \text { for } \alpha>0.6
$$

The meaning of the constraint is that for fractional conversions at which the material has reached the rubbery state and residual stress can build up, the maximum thermal gradient must be kept lower than the thermal gradients achieved during conventional cure schedules. The implementation of the constraint is performed by excluding from the selection and reproduction procedures all individuals which violate it.

The values of the optimization parameters are selected within practically meaningful ranges, i.e. a heat up rate from 0 to $4{ }^{\circ} \mathrm{C} / \mathrm{min}$ and an isothermal temperature from 150 to $190{ }^{\circ} \mathrm{C}$. The algorithm is considered to have converged when the individuals of a generation have very small variation, i.e. the average percentage difference between the members of the population and the average is lower than $0.5 \%$.

The algorithm was executed using three different sets of run parameters. The number of individuals, the length of the string and the average values of the initial population were altered in order to ensure solution independence of the genetic algorithm features. The different sets of parameters are given in Table 2. In all cases the three best individuals were directly passed to the next generation whilst the exchange probability was $40 \%$ and the mutation probability $2 \%$.

Table 2. Genetic algorithm parameter values

\begin{tabular}{l|c|c|c} 
& Case 1 & Case 2 & Case 3 \\
\hline Initial population average heating rate $\left({ }^{\circ} \mathrm{C} / \mathrm{min}\right)$ & 1.5 & 1.5 & 3 \\
\hline Initial population average isothermal temperature $\left({ }^{\circ} \mathrm{C}\right)$ & 160 & 160 & 165 \\
\hline String length & 100 & 40 & 40 \\
\hline Population size & 13 & 9 & 9
\end{tabular}


The convergence of the optimization for the three cases is illustrated in Figs. 4-6. The problem involves only two parameters, thus convergence occurs very fast within fifteen generations in all cases. It can be observed that alteration of the parameters of genetic algorithm does not affect the final result of the optimization. The optimal values found are a heating rate of $3{ }^{\circ} \mathrm{C} / \mathrm{min}$ and an isothermal temperature of $169{ }^{\circ} \mathrm{C}$. Global optimality cannot be ensured, the effectiveness of the optimization can be evaluated by comparing the resulting cure completion time of $64.5 \mathrm{~min}$ with the cure completion time of the conventional thermal profile described previously which was $87.5 \mathrm{~min}$. A reduction in cure cycle time $26 \%$ is achieved, which could have a very significant impact on the total cost of production.

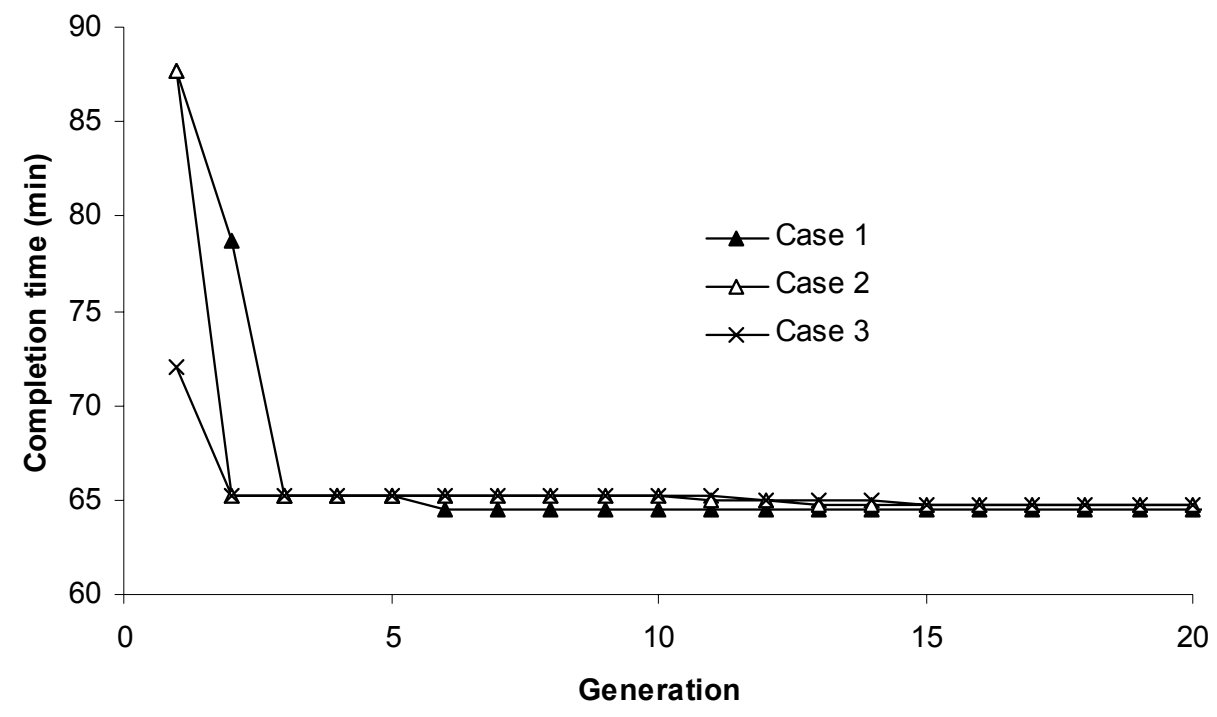

FIGURE 4 Cure completion time vs generation number for the three runs of the genetic algorithm 


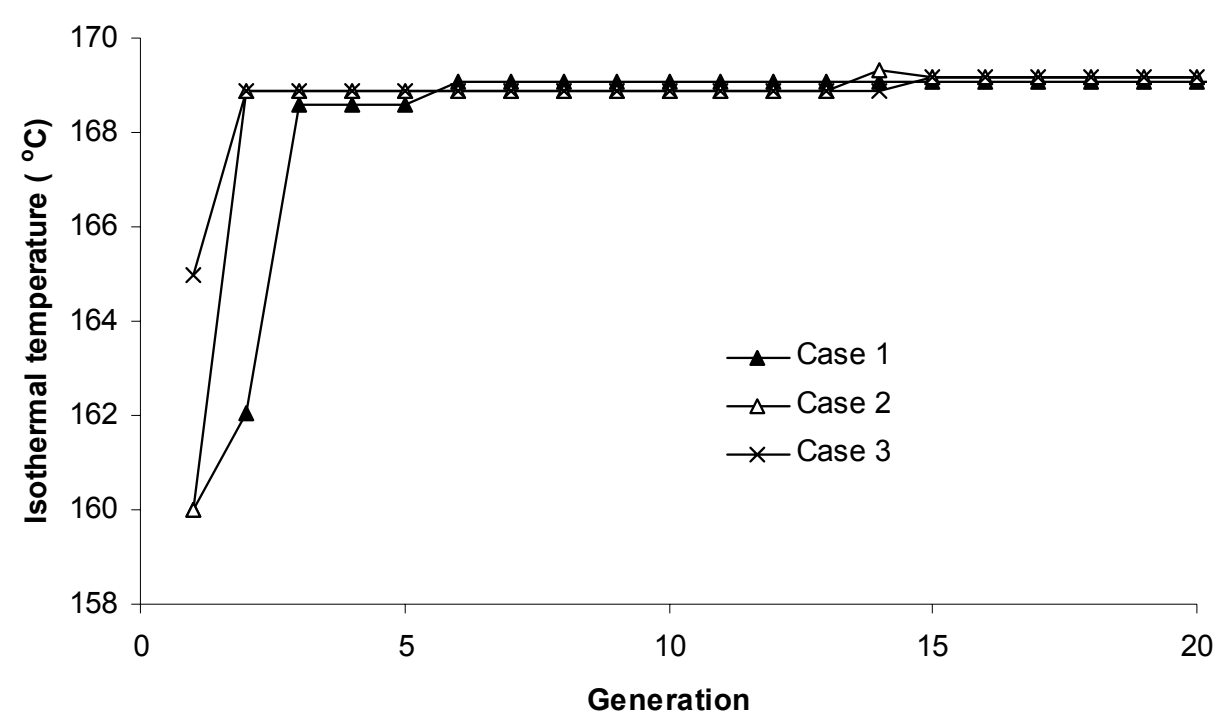

FIGURE 5 Isothermal temperature vs generation number for the three runs of the genetic algorithm

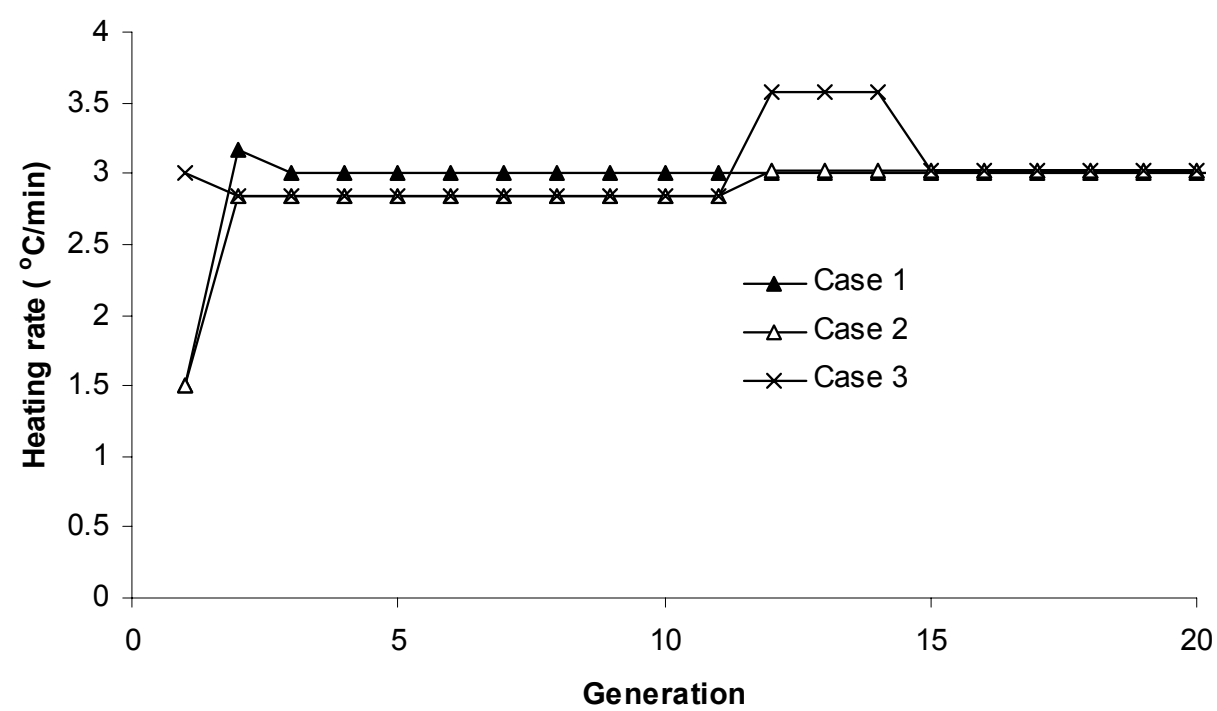

FIGURE 6 Heating rate vs generation number for the three runs of the genetic algorithm

A comparison of the thermal profiles achieved with the optimized process parameters and the conventional cure profile is illustrated in Fig. 7. In the case of the 
conventional cure profile the conversion of $60 \%$ is reached at about $49 \mathrm{~min}$ in the cure whereas in the case of the optimized profile the corresponding time is $36 \mathrm{~min}$. The maximum total temperature gradient from the upper side to the lower side of the curing composite, realized at the end of the heating ramp for both profiles, is $15^{\circ} \mathrm{C}$ during conventional cure and $19{ }^{\circ} \mathrm{C}$ during optimized cure. Consequently, the optimized profile results in higher thermal gradients during heating up and the initial stages of the isothermal segment of the profile, whilst the thermal gradient falls bellow the limit of $2.5^{\circ} \mathrm{C} / \mathrm{mm}$ when the material reaches the rubbery state at $36 \mathrm{~min}$.

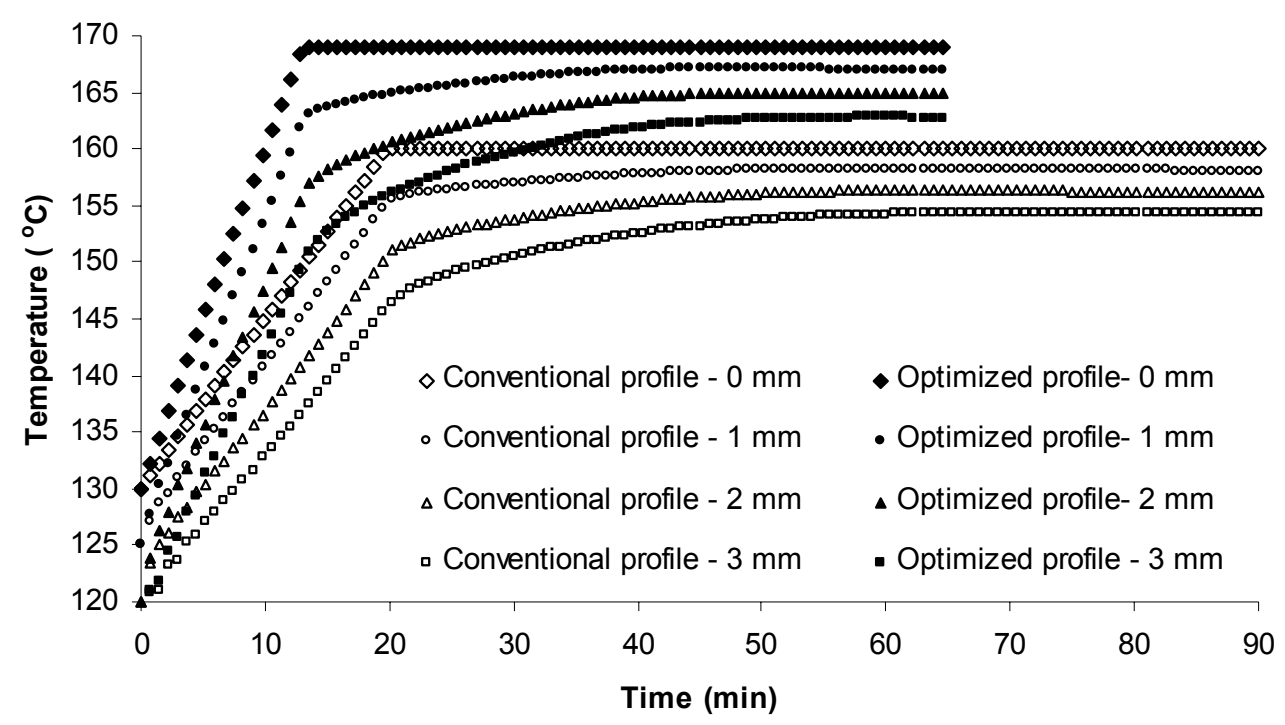

FIGURE 7 Temperature vs time at different locations for the conventional and the optimized cure profile

\section{MODELING-MONITORING INTEGRATION}

The second application of the inversion procedure examined here concerns the use of on-line temperature measurement results in order to determine the dependence of the composite thermal conductivity on the fractional conversion and temperature. Subsequently, the distributions of the temperature and the degree of cure are calculated. 
Thermal conductivity was identified as the critical parameter in the heat transfer simulation as its measurement for a curing thermosetting material presents significant experimental difficulties whilst the sensitivity of the simulation results to its values is high. Fig. 8 illustrates the temperature evolution at mid-thickness of the original simulation and of the simulation when each of the properties involved is increased by $5 \%$ throughout the cure. It can be observed that the sensitivity of the simulation results to specific heat capacity and exothermic heat is low, whereas thermal conductivity and the surface heat transfer coefficient have the most significant influence.

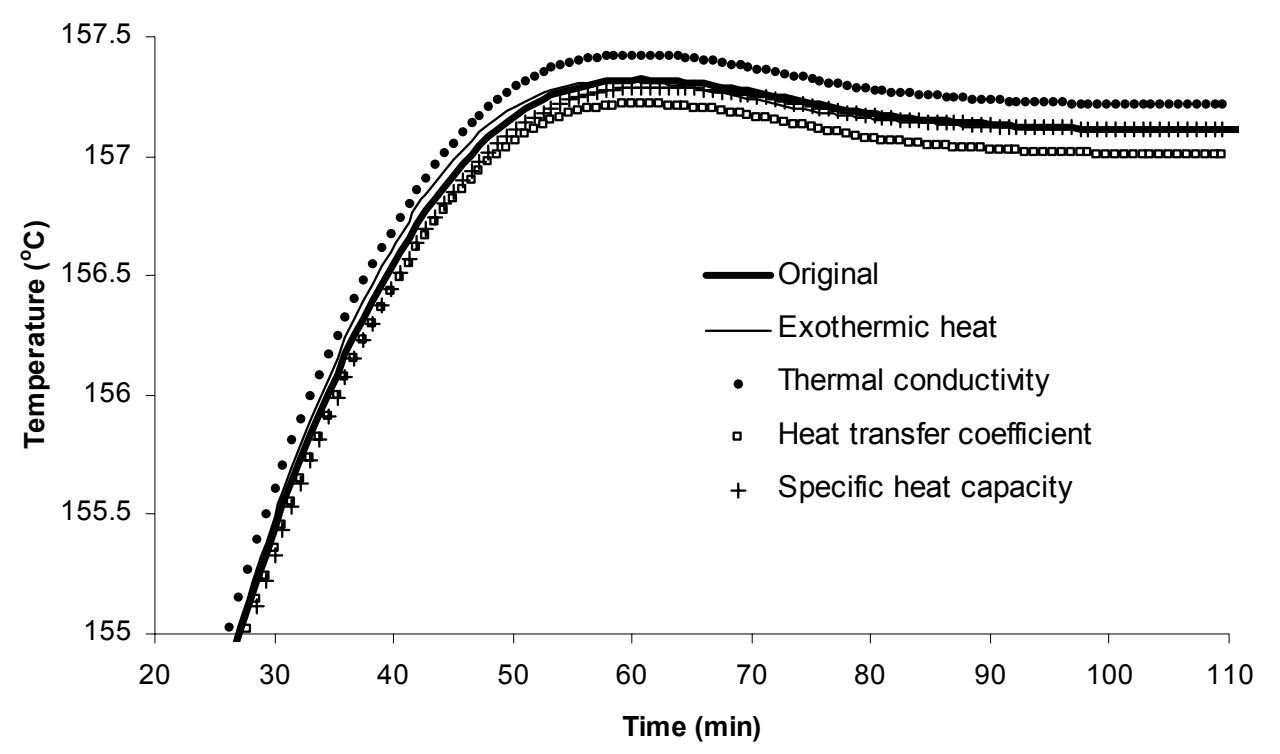

FIGURE 8 Temperature vs time at mid-thickness of the original simulation and of the simulation when each of the thermal properties is increased by $5 \%$

Temperature measurements performed at the mid-depth of a curing carbon/RTM6 composite were used as the target of the genetic algorithm. The variable parameters are the coefficients of a polynomial, which expresses the dependence of the composite thermal conductivity on fractional conversion and temperature as follows:

$$
K=\left(\alpha^{2} P_{1}+\alpha P_{2}+P_{3}\right)\left(T P_{4}+P_{5}\right)
$$


The fitness of an individual is calculated as follows:

$$
\text { Fitness }=1 / \sum_{i=1}^{Q}\left|T_{i}^{\text {mid }}-T_{M}\right|
$$

where $T_{i}^{\text {mid }}$ is the calculated by the model temperature at time step $\mathrm{i}$ at the middle node, $Q$ is the number of time steps and $T_{M_{i}}$ is the temperature measurement at time step i.

The parameters of the finite element model have the values indicated by the convergence study. In the genetic algorithm 26 individuals with a string length of 100 bits were used. Five individuals were passed directly to the next generation, the crossover and mutation probabilities were identical to those used in the thermal profile optimization runs. The range of the five parameters to be estimated was -1 to 1.

The convergence behavior of the algorithm is illustrated in Figs. 9 and 10. The algorithm converges after about 30 generations. Note that the problem in that case involved five parameters and about 900 iterations of the direct model were required for their estimation. A search method equivalent in terms of computational time would have resulted in a accuracy of about 0.5 in the parameter estimation. The solution of the inverse problem is:

$$
K=\left(-0.125 \alpha^{2}+0.117 \alpha-0.094\right)(0.358-0.034 T)
$$

Using this model for the calculation of thermal conductivity, the distributions of temperature and degree of cure can be calculated. Their comparison with the results of the direct simulation, which was shown to be in agreement with the thermal monitoring results utilized here for the inversion, is illustrated in Fig. 11. It can be observed that the monitoring-modeling scheme predicts the global distribution of temperature and degree of cure with satisfactory accuracy. The average error in temperature estimation is $0.29{ }^{\circ} \mathrm{C}$ and in fractional conversion determination 0.0019 . The error in temperature is lower than the accuracy of the direct model whereas the error in fractional conversion estimation is very low due to the fact that the higher 
differences in temperature between the inversion results and the direct model occur at low conversions when the reaction rate is very low.

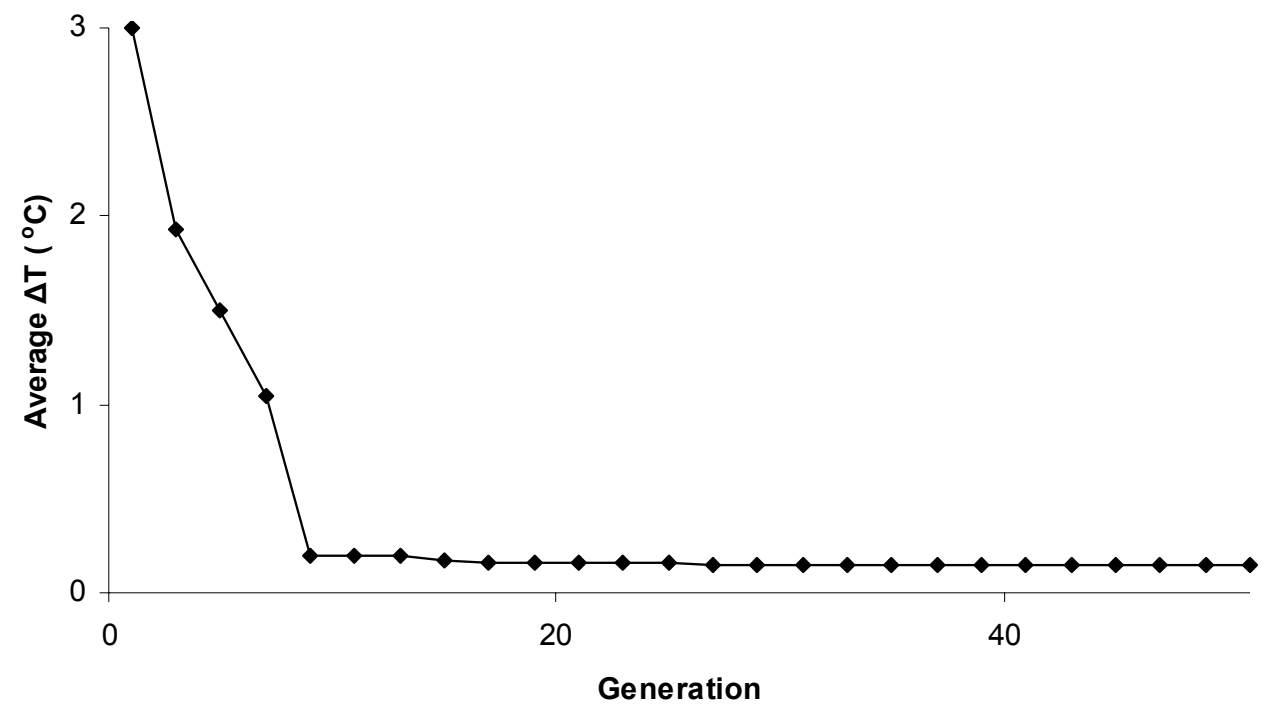

FIGURE 9 Difference between measurement and inverse modeling results vs generation

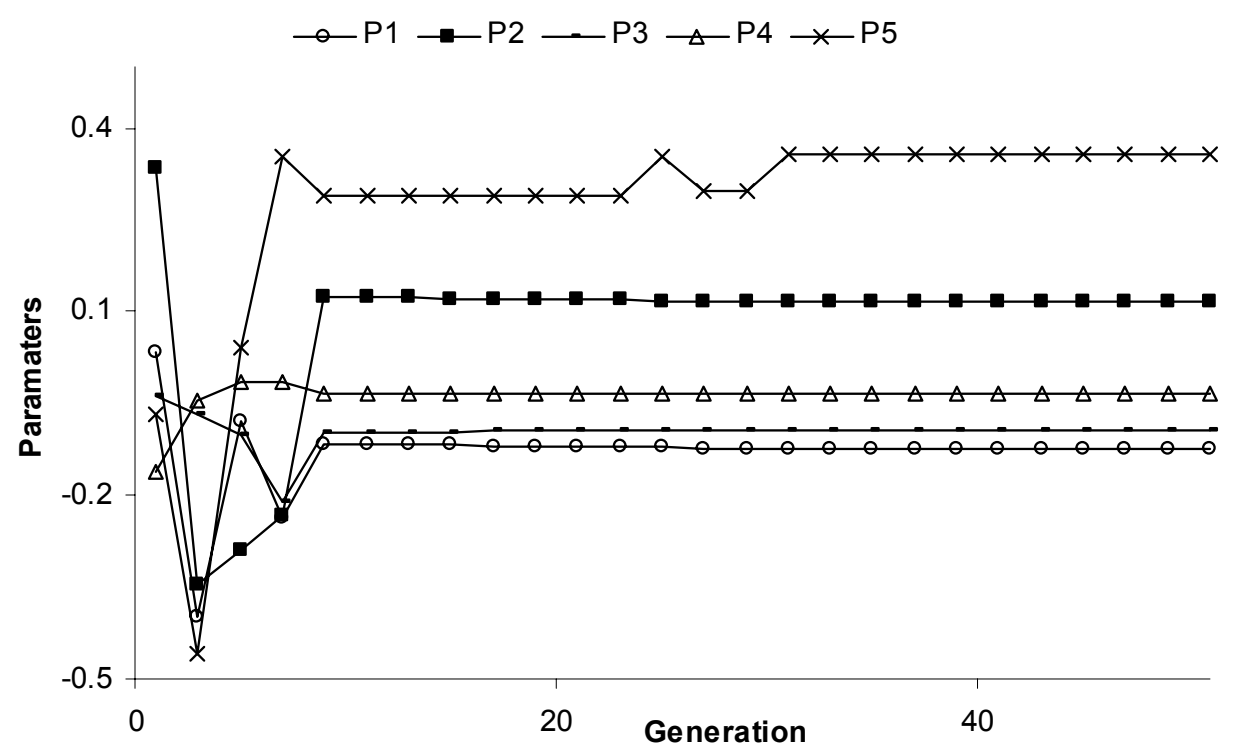

FIGURE 10 Parameters of the thermal conductivity polynomial vs generation number 


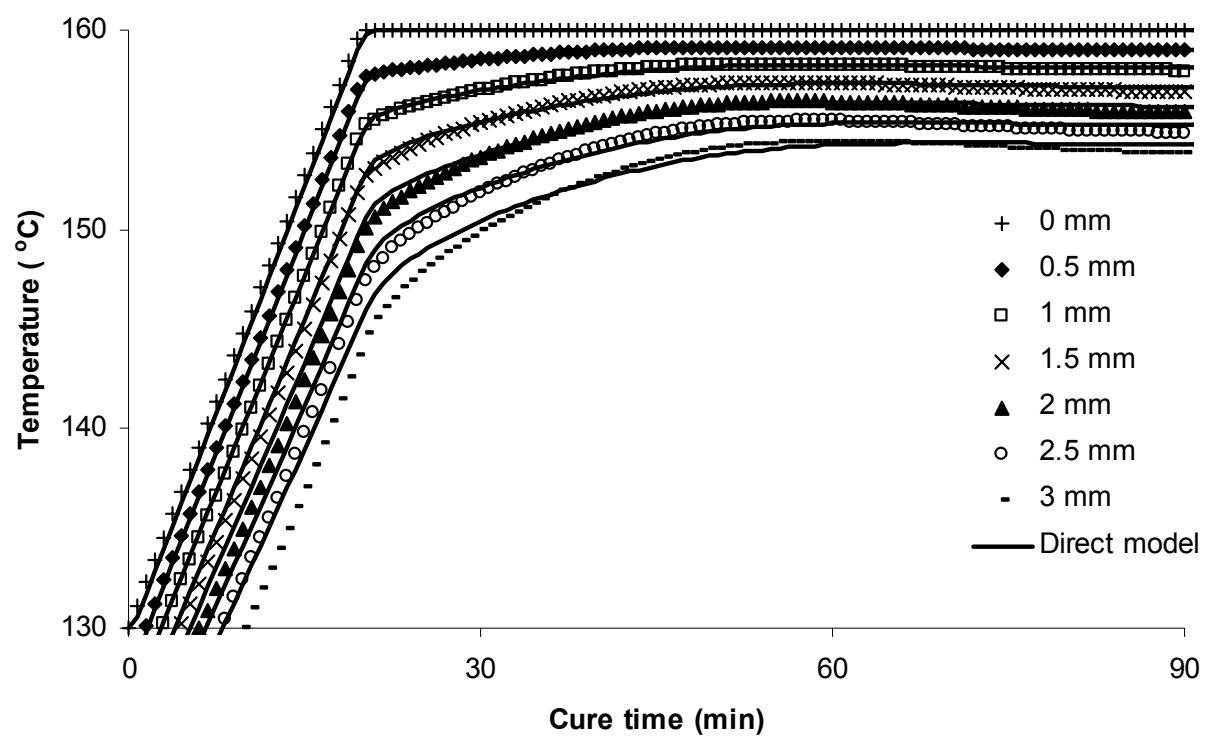

FIGURE 11 Temperature vs cure time at different levels as resulting from the inversion procedure and from the direct model

The results of the sensitivity analysis indicated the high sensitivity of the measured temperature to the heat transfer coefficient. In order to investigate the effect of the heat transfer coefficient value on the estimated parameters of the thermal conductivity model the genetic algorithm was executed using slightly different values of the heat transfer coefficient. Namely the original value of 8.5 $\mathrm{W} / \mathrm{m}^{2} /{ }^{\circ} \mathrm{C}$ was altered to 8.3 and $8.7 \mathrm{~W} / \mathrm{m}^{2} /{ }^{\circ} \mathrm{C}$. The results are given in Table 3. It can be observed that differences up to $6 \%$ in the parameter values are induced by the heat transfer coefficient change and that the average error achieved using a the lower heat transfer coefficient value is lower than the original simulation result. 
Table 3. Influence of the heat transfer coefficient on the estimated parameters

\begin{tabular}{l|c|c|c} 
Heat transfer coefficient $\left(\mathrm{W} / \mathrm{m}^{2} /{ }^{\circ} \mathrm{C}\right)$ & 8.5 & 8.3 & 8.7 \\
\hline $\mathrm{P}_{1}$ & -0.125 & -0.120 & -0.127 \\
\hline $\mathrm{P}_{2}$ & 0.117 & 0.109 & 0.115 \\
\hline $\mathrm{P}_{3}$ & -0.094 & -0.093 & -0.092 \\
\hline $\mathrm{P}_{4}$ & -0.034 & -0.032 & -0.035 \\
\hline $\mathrm{P}_{5}$ & 0.358 & 0.338 & 0.341 \\
\hline Average error $\left({ }^{\circ} \mathrm{C}\right)$ & 0.29 & 0.19 & 0.35
\end{tabular}

\section{CONCLUSIONS}

The inversion procedure based on genetic algorithms presented here can be used for optimization and monitoring-modeling integration in composites manufacturing. Optimal cure schedules with respect to total curing process duration were found for a specific carbon fiber/RTM6 composite component. The monitoring-modeling combined scheme offers the possibility to infer temperature and degree of cure distributions from limited local thermal monitoring signals. Local monitoring results combined with the inversion procedure result in a very accurate estimation of the temperature and degree of cure evolutions during the cure. Both applications of heat transfer inversion can be extended to the case of complex components where two or three-dimensional modeling is required. Application of the monitoring-modeling combined scheme may be limited due to computing time in the case of fast cures, however the majority of advanced composite components are subject to several hours long cure profiles. 


\section{NOMENCLATURE}

$c_{p} \quad$ Specific heat capacity

$h \quad$ Surface heat transfer coefficient

$H_{t o t} \quad$ Total heat of the curing reaction

$\boldsymbol{K} \quad$ Thermal conductivity tensor

$N_{i} \quad$ Interpolation function

$\hat{n} \quad$ Surface vector

$q^{\prime} \quad$ Boundary heat flux

$\hat{\boldsymbol{r}} \quad$ Spatial coordinate

$S_{1} \quad$ Temperature boundary

$S_{2} \quad$ Heat flux boundary

$S_{3} \quad$ Convection boundary

$\tilde{S}_{i j} \quad$ Interface

$T \quad$ Temperature

$T^{\prime} \quad$ Boundary temperature

$T_{\infty} \quad$ Ambient temperature

$t \quad$ Time

$t_{c} \quad$ Time to complete the curing

$v_{f} \quad$ Fiber volume fraction

$\alpha \quad$ Degree of cure

$\Delta t \quad$ Time step

$\theta \quad$ Time discretization parameter

$\rho \quad$ Density of the composite

$\rho_{r} \quad$ Resin density 


\section{REFERENCES}

1. A. C. Loos and G. S. Springer, Curing of epoxy matrix composites, Journal of Composite Materials, 17, 135 (1983)

2. D. M. Gao, F. Trochu and R. Gauvin, Heat transfer analysis of non-isothermal resin transfer molding by the finite element method, Materials and Manufacturing Processes, 10, 57 (1995)

3. R. Gorthala, J. A. Roux and J. G. Vaughan, Resin flow, cure and heat transfer analysis for pultrusion process, Journal of Composite Materials, 28, 486 (1994)

4. S. Y. Lee and G. S. Springer, Filament winding cylinders: I. Process model, Journal of Composite Materials, 24, 1275 (1990)

5. P. I. Karkanas and I. K. Partridge, Cure modeling and monitoring of epoxy/amine resin systems. I. Cure kinetics modeling, Journal of Applied Polymer Science, 77, $1419(2000)$

6. A. A. Skordos and I. K. Partridge, Cure kinetics modelling of epoxy resins using a non-parametric numerical procedure, Polymer Engineering and Science, 41, 793 (2001)

7. G. Lebrun, R. Gauvin and K. N. Kendal, Experimental investigation of resin temperature and pressure during filling and curing in a flat steel, Composites, $A, 27$, 347 (1996)

8. D. E. Kranbuehl, P. Kingsley, S. Hart, G. Hasko, B. Dexter and A. C. Loos, In-situ sensor monitoring and intelligent control of the resin transfer molding process, Polymer Composites, 15, 299 (1994)

9. G. M. Maistros and I. K. Partridge, Monitoring autoclave cure in commercial carbon fibre/epoxy composites Composites, B, 19, 245 (1998)

10. D. L. Woederman, J. K. Spoerre, K. M. Flynn and R. S. Parnas, Cure monitoring of the liquid composite molding process using fiber optic sensors, Polymer Composites, 18, 133 (1997)

11. T. M. Whitney and R. E. Green Jr, Ultrasonics, Cure monitoring of carbon epoxy composites: An application of resonant ultrasound spectroscopy 34,347 (1996) 
12. J. D. Farmer and E. E. Covert, Thermal conductivity of a thermosetting advanced composite during its cure, Journal of Thermophysics and Heat Transfer, 10, 467 (1996)

13. A. A. Skordos, $\mathrm{PhD}$ Thesis, Modelling and monitoring of resin transfer moulding, Cranfield University, UK, 2000

14. T. Yamane, S. Katayama, M. Todoki and I. Hatta, The measurement of thermal conductivity of carbon fibers, in Thermal Conductivity 22 by T. W. Tong, p 313, Technomic Publishing, USA, 1994

15. J. A. Holmberg, Influence of post cure and chemical shrinkage on springback of RTM U-beams, SICOMP Technical Report 97-004, 1997 